\title{
A Study on the Criticism and Self-criticism of "New Social Space" in the Perspective of Social Media Technical Idea
}

\author{
Yan Zou \\ Southwest University \\ School of Journalism \& Communication \\ Chongqing, China \\ 13594093388@163.com
}

\author{
shicha Luo \\ Sichuan University \\ School of Journalism \& Communication \\ Chengdu, China \\ 615056@qq.com
}

\begin{abstract}
Along with the widespread of new media technology in our daily life, plenty of scholars thought highly of the convenience brought by it. On the other hand, such a phenomenon reflected the change of our normal life, thus almost everyone abandoned to rethink the real face of proliferated media technology while enjoying the convenience. Actually, the move of rethinking means a lot under the impetuous environment nowadays. Therefore, this paper chose the new social space formed by new social media technology as the object of study and the new social media technical idea of Sense Technology, Digital Technology and Omnimedia Technology would be the three typical perspective to help the author analyze its social value, more importantly, to rethink the advantages and disadvantages of the regulative development of technology.
\end{abstract}

Keywords-social media; new social space; new media technology

Along with the widespread of new media technology in our daily life, plenty of scholars thought highly of the convenience brought by it. On the other hand, such a phenomenon reflected the change of our normal life, thus almost everyone abandoned to rethink the real face of proliferated media technology while enjoying the convenience. Actually, the move of rethinking means a lot under the impetuous environment nowadays. Therefore, this paper would take the so-called New Social Spaces formed by kinds of new social media technology as examples in order to rethink what fundamental subversions of modern life and existing forms have had brought by the new social media, simultaneously, to do a self-criticism of technical idea which caused the outcome "new".

\section{THE CONTRADICTION BETWEEN NEW SOCIAL SPACE AND SOCIAL INTERCOURSE}

Space is divided into physical space and language symbolic space, furthermore, which can be also described as a cultural ideology and a spatial rhetoric of self-identity. In the book The Production of Space, the author Henri Lefebvre cerebrate the space. He emphasized the social nature of human living space, noting that "the production of relations in the space is an important form of reproduction of social relations."[1] Therefore, people are always wrapped in a complex situation between symbolic space and physical space. Social space is not only closely interrelated to our daily lives, but also determined the level of activity when we do a social intercourse, as well as the quality of public life.

With the immersion of micro-blogging and other social media in our modern life, one certain kind of space has already come into being. In addition, different from "media environment", the concept of "space" includes not only an incredibly realistic mimicry interaction context just like in media environment, more importantly, it emphasizes the relationships in the space as a breakthrough point to do social and cultural criticism. In fact, many scholars had similar logic of analysis in history such as Foucault's "Right Space", Pierre Bourdieu's "Social Space" and Habermas's "Public Space". Therefore, instead of using "media environment", "social networking" and other normal concepts, the author would like to take "Social Space" as the key concept to cut into the social and cultural criticism, so as to express its real association with social life.

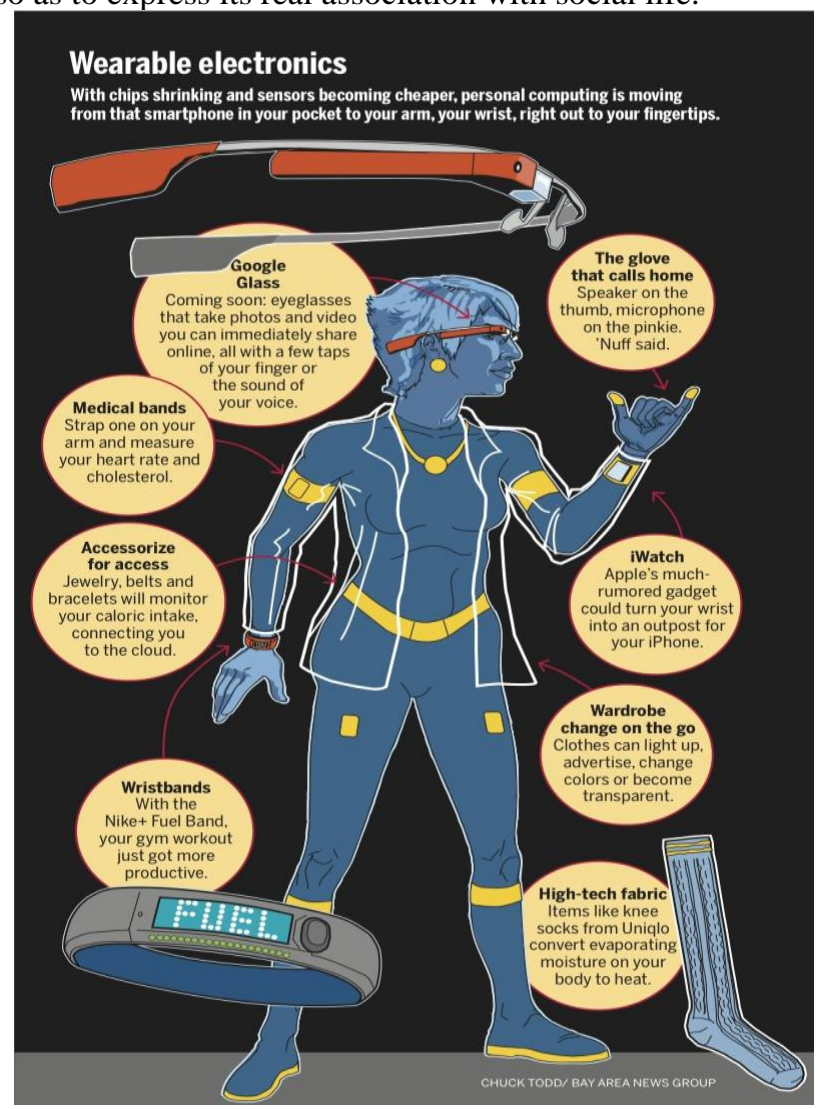

Figure 1. Wearable Electronics[2] 
The proliferated technology gave us a social space just like a work of art. According to Bourdieu's view, such a new social space has its own structure and rules, i.e. the relationships, power relations in the space, etc.; however, according to Simmel Georg's view, social content should be removed, leaving only the pure form. These are two rather significant critical analyses. The former endued social media with more social meaning, while the latter warned us of this virtual technology's giant confusing impact to us from the perspective of the form.

There is no doubt that social media is a platform for the exchange of public opinion, the source of public action, and the oversight department of public interest. But it wastes the user a lot of time every day and spends the public passion of users, of which, I believe, everyone would have the same feeling more or less if only he had a micro-blog. According to HP Labs' survey, compared to the users of Twitter website more concerned about global politics and news time, Sina micro-blog users paid more attention to jokes, pictures, videos and other entertainment information.[3] It can be seen that the sense of participation and public enthusiasm brought by numerous kinds of social media technology were only the moon reflected in the water, or flowers in the mirror, which creates a false picture of the social, thus hindering the real socializing.

The famous professor Wang Xiaoming delivered a prose to Frontiers, lamenting the disappearance of "square" which is a very typical "space" in city. There are only some commercial pedestrian streets or shopping centers nowadays, the "square" which has the traditional functions such as association, assembly and so on gradually disappeared. And the popular concept "global village" put forwarded by Marshall McLuhan, as is often used to explain how the new social media makes the world become near at hand, the correct meaning had been ignored, which is the future of human race is Re-tribalisation and then live a face to face social life for real like living in a village back again, returning to those disappearing social space in the past. Perhaps this is the correct meaning of real social intercourse.

\section{THE CRITICISM OF NEW SOCIAL SPACE WITH NEW SOCIAL MEDIA TECHNOLOGY}

\section{A. The Social Space of "Humanization" Formed by Sense Technology}

Sensory technology put forwarded as some concept of "technology" is a strange statement, but the similar concepts such as "new sense" and "multi-sensory" had lots of use in the field of product design like packaging and other fields. Sense Organ means "the stimuli organs to feel things outside, i.e. eyes, ears, nose, tongue, skin and other receptors and the corresponding region of the cerebral cortex and nerve transmission system, etc., collectively referred to as sensory systems"[4]. And Sense Technology is a kind of technology which chooses variable sensory language to code and decode, so as to express in an appropriate way in order to make products better meet the user's sensory needs.
It is quite normal and successful that Sense Technology applied to the social media. Micro-blog and Wechat, the most popular two applications, both are the models of multi-sensory technology integration. Wechat, it can be seen from the name to some extent, is able to be used to voice chat, share photos, voice recording and voice prompts. It is not only one kind of social media, but also a living tool. To be precise, Wechat which can be described as a tool for completely IP-based message sharing took the place of the traditional telecommunications business SMS and MMS gateways, as well as voicemail server's SMS (text messaging), MMS (multimedia message service) and VMS (voice mail) services.[5] It is understandable that Wechat, such a concentration of communication services, had a good group of fans. In the meantime, Wechat helps users to imagine a broader new social space which the author summed up as "humanization" for the following reasons.

1) More sense organs of human mobilized in social space of humanization

Karl Marx believed that "the formation of the five senses is the result of the past whole history of the world." Not only the formation of history depends on the general perception of sensory experience, in fact, but also the perception of reality requires all kinds of sense experience, which can be understood as the representation "face to face" communication and the exertion of "human nature". Wechat found the absence of "hearing" sensory experience and did a lot of useful attempt in the field of sense technology, which finally make Wechat become a social media of 300 million users.

2) Humanized design concept under social space of humanization

Particularly, humanized design concept includes.

Firstly, the audience's position which is "not for minority" as the threshold of the social space is much humanized.

When the "circle of friends" of Wechat was first introduced, this design has been criticized for doing sucks and it cannot be compared to Path. Zhang Xiaolong, the Vice President of Tencent, principal of Wechat team responded like this, "what on earth do you want, whether a kind of electric product for minority or one even for the migrant workers?"[6] "Not for minority" was the first principle of Wechat design team. Such a design concept, in addition, can also be seen in the function of text publishing in the "circle of friends". In fact, the "circle of friends" in the first version does not have a development plan of the text publishing function. It is more precise to say that the purpose is to stop users from sending texts. As for the Wechat team it seems to be not easy to write on the phone and the text is very easy to separate the levels of writing, it would be easy for the people who were good at it to put down the average person immediately, which contradict the "not for minority" audience's position. However, different from text publishing, everyone should be capable of taking pictures and photographs. And the pictures were only saved chronologically without any sort of grouping. Their realistic meaning would make photos' degree of refinement less 
important and ignore the process of PS.

In addition, Wechat contacts do not set up groups, only providing the most simple searching service, thus there would not be too many social theory to distract us when we use Wechat, because what Wechat sees is "all kinds of relationships will naturally develop"[7]. And "humanized" communication is the base of the evolution of the relationship. Therefore, the design concept of Wechat focused on the humanized communication clearly and directly.

Secondly, I/O platform as the inside of the social space is the spiritual core of Wechat technology.

The so-called I / O platform is the Input / Output platform. There are two technical designs might explain how did I/O platform transfer abstraction into simplicity, which is "shake" and "sweep". "Shake" and "sweep", which is no more than the literal meaning, is actually a way to lower the threshold of user-generated information production to the extreme. And it reflects the figurative scene when the user input or output some data. Wechat will be able to deal with the data and give feedback after the information processing. This design concept in social media such as Wechat is one kind of "respect" to the "face to face" communication the real social intercourse.

\section{B. The Social Space of "Big Data" Formed by Digital Technology}

Big Data is a new thing based on the development of digital technology and the acquisition of data-oriented thinking logic. Professor Victor Meyer Schoenberg working in Internet Institute of Oxford Networking Academy, known as "the prophet of Big Data Era", gives a general description of the Big Data. Particularly, Big Data "do not deal with any random sample, but the sum of it". Moreover, it is not precise, but disordered" and "is not causation, but correlation". Jump to be conclusion, Big Data is a way of thinking firstly that the pursuit of greater, more and better data and one technology mainly used for prediction secondly.

No matter people are aware or not, our social space is indeed being digitized, and develops toward Big Data. "The thinking of Data-oriented is the spiritual core of many social networking companies. Social networking platform not only provides us with a space for finding friends and maintaining friendships, the same as colleague relations, but also is capable to transfer the intangible elements of our daily lives to data for totally new uses."[8] Particularly, the "social graph" of Facebook is the result of social relationships' digitized processing. And Twitter digitized people's thoughts, emotions and communication successfully. LinkedIn also did some work in digitizing the data of the users' past long experience. Besides, there are also many domestic social media learning from those external experiences. For instance, the so-called "micro index" of Sina micro-blog is the digitize processing of the micro-blog users' social skills and speech impacts. The so-called "timeline" of Renren is also digitized processing of users' past experiences on social media. The application "recommendation" of Douban, considered as the most valuable, is owing to Big Data too.

These primary attempts of Big Data had received considerable commercial return. In the future, the social media company who has some kinds, the more the better, of Big Data could easily grasp the prospects of all sectors of society and be aware of almost all of the dynamic information from every corner of society. This would be a new social space, in which people's relationships are no longer ambiguous. Furthermore, the traditional acquaintance society and the culture of drinking will be impacted. Anyway, this is one technical idea of commercial value with great potential at least.

\section{The Social Space of "Qualitative" Formed by Omnimedia Technology}

Omnimedia as jargon closely integrated with practice has rich connotations. As for the practitioner in media industry, the so-called omnimedia times gave new challenge to them. And it can be seen from the name of "omnimedia times", there must be a considerable degree of universality and enthusiasm in the users of such "times". Then as for the public, it is also a new life in new times.

The formation of media-controlled society is the previous stage of omnimedia times. From McLuhan's "global village" concept to Negroponte's "Being Digital" statement, we can conclude that technology-driven media changes affect the most basic human lifestyle directly. Until the formation of the media-controlled society, human beings had a strong motivation of information possession and controlling finally. And the new social media, most of the time, tend to intervene or simply replace the real-life human interaction. Therefore, to clarify the formation process of the media-controlled society helps the reader to understand the changes of social space caused by Omnimedia Technology.

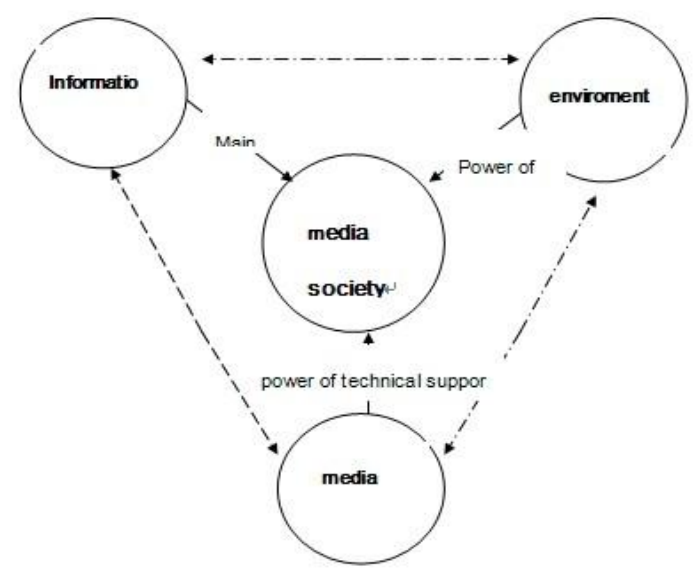

Figure 2. Three Logical Understanding on the Formation of Media-controlled Society[9]

People's relationships and the social space's environment had been redefined in the media-controlled society. Particularly, the philosophy professor Jos de Mul working in Erasmus University in Netherlands Rotterdam 
put forward with a concept "virtual anthropology" in his book Cyber Space Odyssey. In other words, he provided an anthropological perspective for the audience behavior research in the cyber space.

"When a new form of media appears the older ones usually do not die, on the other hand, they would continue to develop and adapt to the situation."[10] Omnimedia as a new form of media contains multiple media forms and integrate a variety of means of communication thus realized the total perception of human information, which is, undoubtedly, the improvement of quality compared to the original social space.

\section{THE SELF-CRITICISM OF NEW SOCIAL MEDIA TECHNOLOGY}

\section{A. The "Asocial" Issue Caused by Humanized Sense Technology}

The future of Sense Technology must be more and more real. The biggest paradox is that, since we already have a real social situation, do we need an analog version of it, although it is almost real. It is no doubt that Technology, especially social media technology, can help us to cope with various problems in the future. For instance, to turn complex problems into simple and to make lengthy things convenient, etc. However, the premise of those advantages is one certain technology must be designed to play a certain role. On the contrary, in the development trends of Sense Technology, it seems that those advantages are extra, and the stimulus to the sense organ is the only thing users care about under social situations of analog version. Problem is the significance of real social situations such as assembly and association and other public activities is of direct benefit to human society. However, the further development of sense technology will replace the effort-wasted real social intercourse inevitably, which also discouraged the possible significance of real social situations.

April 4, 2013, the Professor of School of Journalism \& Communication in Nanjing University @Du Junfei said on his micro-blog, "Years later, people come to the cemetery of the New Media bustlingly... someone asked how to find the tomb of micro-blog and Wechat? The grave guard answered leisurely, the one with a crowd and without a speaker is micro-blog and the one with a crowd who is always talking to themselves is Wechat. Mark Zuckerberg the CEO of Facebook also said that the next social media trend is "sharing within small groups" [11] in a recent interview. It is worth of positive reflection that the "asocial" issue of Sense technology. The spiritual core of social intercourse is not the real based on sense organ but the practical significance of social behavior itself.

\section{B. The "Utility" Issue Caused by Digitized Processing with Big Data}

"Utility" is always used to describe a deliberate calculating characteristic in our daily life. The "utilitarianism" in Western philosophy, although only focus on the results and regardless of motivation, but compared to the utility in everyday life, both of them are "countable",
Utilitarianism requires that people should behave in order to achieve the greatest good, and the calculation of the greatest good lies on the sum of biter and sweet feelings of each individual involved. Although such calculation do not equal to the work of digitized processing, it is quite alike that both of them focused on data.

The most significant feature of Digitized Processing is the integrity of object's memory and the convenience for extract. Huge amount of digital information might cause the so-called "decontextualization" and "recontextualization" when extracting useful information [12]. In short, there is no reference to trace the source of the information when taking advantage of digital information, unless it is clearly marked in advance, which is not possible. For instance, a cited network image and a certain piece of tone borrowed from another song, etc. Digital information has been encoding and decoding for many times, then people will miss the key point of information. The worse is it is almost impossible to change, the first important feature of Big Data is the sum of all data samples as the basis of calculation. It seems that there was a black box separated the calculation and the conclusion. And human beings are highly subjective and emotional creatures, even it is not possible to calculate the degree of "social skills". But in the future, facing so much data information, human beings probably have to focus on the data only. It is easy to connect the basic opinions of utilitarianism with such "utility" issue discussed above.

When all subjective, emotional, abstract things can be calculated into some data, the entire social space will be filled with utility culture, although the purpose from the very beginning may be good.

\section{3.3 The "Similarity" Issue Caused by INFORMATION Dependence of Omnimedia Technology}

There has always been great tension between the change of media technology and social cultures. With the digital revolution in contemporary society, human beings stepped into the digital media society gradually. Omnimedia Technology has adjusted both in concept and in practice of digital information in order to guarantee the quality of relationships. However, the gross of data and complicated categories produced by Omnimedia Technology is conceivable. Although there are possibilities of technological convergence in theory, however, it is often to share the same multimedia material from the same copy of the original case, the road of material integration yet to explore. On the other hand, we also need to be vigilant when people depended on the information deeper and deeper, but when individual needs are not being met in a wide range of social life, the pursuit of omnimedia information become urgent. After all, people's relationships are different, and a good social space should not only have high-quality information, more importantly, should deliver the feelings of whether cold or warm, etc. of social life. Otherwise, human beings will not be that different from robots. 


\section{CONCLUSION}

Contemporary technological civilization surviving crisis as well as the spiritual crisis of nihilism has become a common concern of writers and scholars through the age. However, the author does not agree to criticize technological civilization easily. To be frank, the rapid development of science and technology often be considered as being driven the inevitable global process of modernization, but in the final analysis, human indeed harvested a lot from the technological civilization development effectively. As for an individual, the using condition is affected by the stability of the macro-social environment directly. Therefore, to observe subtle changes when the new social media technology impact the social space, and live a real life poetically together with technology, which is how we can avoid the incomprehensible but real modern anxiety.

In fact, the question that whether can the poetic elements be put into new social media technology mostly determined by the poetic thinking in users of technology but not ability of technology itself. After the above analysis, it is easy to see the future development of new social media technologies always focused on the user in general and alter the logical starting point because of the audience group. In other words, the problems of human beings cannot depend on technology civilization completely. That is to say technology is only the macro-social background, even in history. And the only way out is to be aware of the defect of "technology versus Society" simple thinking, then it might be possible to connect people with technology poetically.

\section{ACKNOWLEDGMENT}

Supported by "the effectiveness research about the public service advertisement in the context of new media"

NO: SWU2120121017

\section{REFERENCES}

[1] Wang Dun, "The hearing flow in the cultural space: historical and social," J. Literature \& Art Studies. 2011.5.

[2] Information gragh: wearable electronics, J. San Jos Mercury News,2013.3.18.http://www.mercurynews.com/business/ci_2281806 8/info graphic-wearable-electronics-what-apps-do-you-have

[3] Wang Weijia, Yang Lijuan, "'Wu Ying Case' and the party spirit of micro-blog intellectuals," J. Open Times, 2012.5.

[4] Lei Ming, Li Li, "Sensory experience and extension in advertisement," J. Market Modernization, 2007.7.

[5] $36 \mathrm{Kr}$, Chang Liang, "The telecommunications elements in Wechat," http://www.36kr.com/p/202562.html

[6] Salon for internet analysis, "What is Wechat?" http://www.techxue.com/portal.php?mod=view\&aid=3481

[7] Salon for internet analysis, "What is Wechat?" http://www.techxue.com/portal.php?mod=view\&aid=3481

[8] Viktor Mayer-Schönberger, Sheng Yangyan, Zhou Tao translation, "Big Data:A Revolution That Will Transform How We Live, Work, and Think," M. Zhejiang People's Publishing House, 2013.119.

[9] Zhang Xiaofeng, "Three logic of the formation of media-controlled society, ” J. Modern Communication, 2010.7.

[10] Roger Fidle, Ming Anxiang translation, "Media Morphosis: understanding new media," Huaxia Publishing House, 2000.19.

[11] Huxiu, Zuckerberg, "The next big trend is shared within small groups," http://www.huxiu.com/article/12691/1.html

[12] Viktor Mayer-Schönberger, Yuan Jie translation, "Delete: The Virtue of Forgetting in the Digital Age," M. Zhejiang People's Publishing House, 2013.114. 